\title{
Respuestas morfo-fisiológicas de plantas de Lithraea caustica (Anacardiaceae) sometidas a restricción hídrica controlada
}

\author{
Morpho-physiological responses of Lithraea caustica plants (Anacardiaceae) \\ under restricted water conditions
}

\author{
Karen Peña-Rojas a*, Sergio Donoso a, Cristian Pacheco a, Alejandro Riquelme ${ }^{\text {b }}$, \\ Rodrigo Gangas a, Antonella Guajardo a, Sergio Durán ${ }^{\text {a }}$

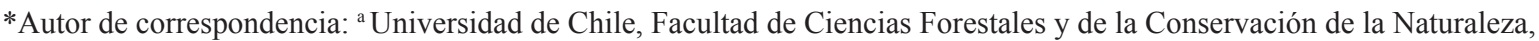 \\ Santa Rosa \# 11.315, Santiago, Chile, kpena@uchile.cl \\ ${ }^{\mathrm{b}}$ Universidad de Chile, Facultad de Ciencias Agronómicas, Departamento de Producción Agrícola, Santiago, Chile.
}

\section{SUMMARY}

\begin{abstract}
Lithraea caustica (litre) is an endemic tree species of Chile that grows in a Mediterranean climate, characterized by a summer with low water availability in the soil, high solar radiation and high temperatures. In these conditions, litre should have physiological mechanisms to survive under restricted water conditions. To investigate these mechanisms we performed an experiment under natural summer conditions. The plants were assigned to three different treatments: Permanent irrigation, moderate water restriction and severe water restriction. During the study, we evaluated predawn leaf water potential and predawn leaf water relative content, photosynthesis, gas exchange and chlorophyll fluorescence. Additionally, we evaluated the growth and accumulation of biomass. Under restricted water conditions, litre developed the osmotic adjustment mechanism, presenting a high cellular turgor and a leaf water relative content over $80 \%$. This strategy allowed litre to tolerate the low soil water contents observed during the phase of maximum water restriction. It also repaired damages caused during the photoinhibition period observed at noon, with values of $\mathrm{F}_{\mathrm{v}} / \mathrm{F}_{\mathrm{m}}$ always equal to or higher than 0.8 . This study provides valuable information of litre attributes that could be applied in future scenarios of reforestation and climate change in Mediterranean areas.
\end{abstract}

Key words: litre, Mediterranean forest, drought, osmotic adjustment, photoinhibition.

\section{RESUMEN}

Lithraea caustica (litre) es una especie arbórea endémica de Chile que se desarrolla en un clima de tipo mediterráneo, caracterizado por presentar una estación estival con baja disponibilidad hídrica del suelo, elevada radiación solar y altas temperaturas. Bajo estas condiciones, litre debe desarrollar respuestas ecofisiológicas que le permitan sobrellevar períodos de restricción hídrica. Con el objetivo de conocer dichas respuestas, se estableció un ensayo de restricción hídrica controlada bajo condiciones naturales de verano. Las plantas se sometieron a tres tratamientos: riego permanente, restricción hídrica moderada y restricción hídrica severa. Durante el estudio se evaluaron variables hídricas (potencial hídrico foliar a pre-alba y contenido hídrico relativo foliar a pre-alba), fotosíntesis, intercambio gaseoso y fluorescencia de las clorofilas. Adicionalmente, se evaluó el crecimiento y acumulación de biomasa. Bajo condiciones de restricción hídrica, litre presentó el mecanismo de ajuste osmótico, permitiendo mantener un alto turgor celular y un contenido hídrico foliar sobre $80 \%$. Esta estrategia permitió a litre tolerar los bajos contenidos hídricos de sustrato observados durante la fase de mayor restricción hídrica. También fue capaz de reparar los daños provocados durante el periodo de fotoinhibición observado a mediodía, con valores de $\mathrm{F}_{\mathrm{v}} / \mathrm{F}_{\mathrm{m}}$ siempre igual o superior a 0,8 . Se entrega información valiosa sobre los atributos de litre, posible de aplicar en futuros escenarios de reforestación y cambio climático en zonas mediterráneas.

Palabras clave: litre, bosque mediterráneo, sequía, ajuste osmótico, fotoinhibición.

\section{INTRODUCCIÓN}

Lithraea caustica Hook. et Arn. (litre), es un árbol siempreverde endémico de Chile, perteneciente a la familia Anacardiaceae. Se distribuye ampliamente por el valle central y la Cordillera de la Costa entre los $30^{\circ}$ y $39^{\circ}$ de latitud sur, mientras que por la Cordillera de Los Andes sólo solo se circunscribe a los contrafuertes cordilleranos entre las ciudades de Santiago y San Fernando (Cabello y Donoso 2006). Es un componente principal en el bosque esclerófilo mixto, ocupando zonas altas y de media altitud, en exposiciones secas y cálidas [asociación Quillaja saponaria Poir. (quillay) - Lithraea caustica Hook. et Arn. (litre)] o exposiciones húmedas y sombrías [asociación Cryptocarya 
alba (Molina) Looser (peumo) - Lithraea caustica] en la región mediterránea (Donoso 1982). Este autor también le describe como especie acompañante en los espinales de Acacia caven (Mol.) Mol. (espino), matorrales más xéricos que han sido considerados como etapas pioneras de la sucesión secundaria (Root-Bernstein et al. 2017).

Litre se desarrolla bajo el clima de tipo mediterráneo, caracterizado por presentar una fuerte estacionalidad, con precipitaciones concentradas principalmente en invierno, y un periodo con baja disponibilidad hídrica del suelo, elevada radiación solar y altas temperaturas durante el verano, cuya duración e intensidad disminuyen en latitud (Luebert y Pliscoff 2006). Durante el periodo estival, las condiciones ambientales favorecen la aparición de múltiples estreses en las plantas y se ha observado que diferentes especies que crecen en un mismo hábitat, desarrollan distintas estrategias para mantener el turgor celular adecuado (Donoso et al. 2011, 2015).

En condiciones de déficit hídrico, las plantas experimentan un descenso en sus parámetros hídricos internos en respuesta a la menor disponibilidad de agua en el perfil de suelo, lo cual repercute en un detrimento general de la actividad fisiológica y fotosintética. Bajo estas condiciones, el aparato fotosintético se predispone a sufrir inhibición de la fotosíntesis causada por exceso de radiación (fotoinhibición), debido a potenciales hídricos bajos la fotosíntesis puede ser alterada por efectos no estomáticos, principalmente por una reducción en la actividad de los fotosistemas II (FSII) y en las reacciones de transferencia de electrones (Peña-Rojas et al. 2004, Fleck et al. 2010, Ceacero et al. 2012, Flexas et al. 2014).

Algunas plantas sometidas a déficit hídrico desarrollan mecanismos fisiológicos de tolerancia a la deshidratación como el ajuste osmótico o el ajuste elástico, que les permiten sobrellevar este estrés (Fan et al. 1994). Ambos mecanismos permiten mantener el suministro de agua a los tejidos de la planta, el turgor celular, los procesos fisiológicos, la sobrevivencia y el crecimiento bajo condiciones de restricción hídrica. Al respecto, diversos estudios han descrito el comportamiento de varias especies mediterráneas europeas ante un escenario de restricción hídrica (Peña-Rojas et al. 2004, Gallé et al. 2007, Fleck et al. 2010, Flexas et al. 2014). Sin embargo, aún es exigua la información sobre las respuestas fisiológicas de los componentes florísticos más importantes del bosque mediterráneo chileno.

Basándose en la persistencia de litre en condiciones secas del bosque esclerófilo, se plantea como hipótesis de trabajo que esta especie cuenta con mecanismos de tolerancia al estrés hídrico que le permiten adaptarse al estrés estival. El objetivo del presente trabajo es estudiar la respuesta morfo-fisiológica de plantas de litre sometidas a restricción hídrica moderada y severa. Se discuten las posibles implicaciones sobre el establecimiento de la especie en el campo, información que puede ser útil en futuros programas de reforestación o restauración bajo condiciones mediterráneas.

\section{MÉTODOS}

Sitio de estudio y diseño experimental. El estudio se desarrolló en el campo experimental del vivero Antumapu de la Universidad de Chile, entre diciembre y marzo ( $33^{\circ} 34^{\prime} \mathrm{S}$ y $70^{\circ} 37^{\prime} \mathrm{O}$; $420 \mathrm{~m}$ de altitud), bajo condiciones ambientales naturales de verano. Durante el periodo de estudio, se presentaron condiciones normales de temperatura: la media máxima alcanzó los $28,8^{\circ} \mathrm{C}$ y la media mínima los $11,6^{\circ} \mathrm{C}$. Además, precipitó en cuatro oportunidades, siendo más importante la precipitación de marzo, con $12 \mathrm{~mm}$. Sin embargo, ninguna de ellas tuvo implicancia en el estado hídrico de los individuos, puesto que se procuró cubrir las plantas sometidas a restricción hídrica, en cada oportunidad de precipitación.

Un mes antes del inicio del ensayo, se seleccionaron un total de 130 plantas de dos años de edad de la especie L. caustica, las que fueron dispuestas en macetas de nueve litros de capacidad utilizando una mezcla de suelo del lugar (de origen aluvial y de la serie Santiago), de textura franco arenosa, perlita y arena en una proporción 4:3:3, respectivamente. Adicionalmente, se complementó el sustrato con superfosfato triple y urea $\left(1,20\right.$ y $\left.0,32 \mathrm{~g} \mathrm{~L}^{-1}\right)$. Las plantas fueron mantenidas bajo constante irrigación (contenido hídrico del sustrato (CHS) superior a $80 \%$ ), con el fin de homogenizar la condición fisiológica e hídrica de los individuos, y evitar el efecto de estrés posterior al trasplante.

Del total de plantas disponibles, se seleccionó un grupo de 30 plantas para conformar el tratamiento con aporte hídrico permanente (tratamiento control = TC), 40 plantas para el tratamiento de restricción hídrica moderada (TM) y 50 plantas para el tratamiento de restricción hídrica severa (TS). El monitoreo y control de la condición hídrica en los tres tratamientos se efectuó en función del contenido hídrico del sustrato [1]:

$$
C H S(\%)=\frac{P f s-P s s}{P h s-P s s} \times 100
$$

Donde $C H S=$ contenido hídrico del sustrato (\%), $P f s=$ peso fresco del sustrato tomado al momento del control de peso $(\mathrm{kg}) ; P h s=$ peso hidratado del sustrato $(\mathrm{kg})$, obtenido una vez drenada el agua gravitacional, y PsS = peso seco del sustrato $(\mathrm{kg})$, el que se obtuvo después de secar el sustrato en una estufa de aire forzado a $105^{\circ} \mathrm{C}$ hasta obtener un peso constante.

El monitoreo de la variable se hizo periódicamente tomando una muestra aleatoria de 10 individuos de cada tratamiento, midiendo el peso fresco del sustrato.

El diseño del ensayo consideró que las plantas del tratamiento control se mantuvieran durante todo el ensayo con valores de CHS en torno al $80 \%$. Por su parte, para los tratamientos con restricción hídrica se dispuso una reducción paulatina del CHS a partir del día 12, condición 
que se mantuvo hasta el día 60 , momento en cual ambos tratamientos con restricción hídrica deberían llegar a niveles estables de CHS ( $30 \%$ en el tratamiento con restricción hídrica moderada y $20 \%$ en el tratamiento con restricción hídrica severa). Ambos tratamientos con restricción hídrica se mantuvieron bajo esta condición durante 36 días, para luego nuevamente ser rehidratados (día 96), hasta alcanzar valores de CHS similares a los presentados en el tratamiento control.

Evaluación de variables hídricas. Se hicieron evaluaciones del potencial hídrico foliar a pre-alba $(\Psi)$ y del contenido hídrico relativo foliar a pre-alba $\left(\mathrm{CHR}_{\mathrm{a}}\right)$. El potencial hídrico foliar a pre-alba fue determinado con una bomba de presión PMS Instruments modelo 1000 (Corvallis, OR), mientras que para el cálculo del $\mathrm{CHR}_{\mathrm{a}}$ se siguieron los procedimientos descritos por Galmés et al. (2007). Las mediciones se efectuaron a partir de una muestra aleatoria de ocho individuos de cada tratamiento, procurando que estas provinieran de brotes ubicados en el tercio superior de cada una de las plantas, con características similares en cuanto a número, madurez, tamaño y sanidad. La oportunidad de medición se definió en función del gradiente de CHS obtenido durante el ensayo según lo indicado en el cuadro 1 .

Con parte del material utilizado para medir $\Psi_{\mathrm{a}}$ y $\mathrm{CHR}_{\mathrm{a}}$, se elaboraron curvas de presión-volumen (Tyree y Richter 1981, Corcuera et al. 2002). Las curvas permitieron estimar los parámetros: potencial osmótico a cero turgor $\left(\pi_{0}\right)$ y a turgor máximo $\left(\pi_{100}\right)$, módulo de elasticidad de la pared celular $(\varepsilon)$, potencial de presión a turgor máximo $\left(\Psi_{\mathrm{P} 100}\right)$ y contenido hídrico relativo a cero turgor $\left(\mathrm{CHR}_{0}\right)$. La es-

Cuadro 1. Cronograma de medición para la evaluación de variables hídricas.

Measurement schedule for the evaluation of water variables.

\begin{tabular}{cccc}
\hline $\begin{array}{c}\text { Día de } \\
\text { evaluación }\end{array}$ & $\begin{array}{c}\text { CHS en TC } \\
(\%)\end{array}$ & $\begin{array}{c}\text { CHS en TM } \\
(\%)\end{array}$ & $\begin{array}{c}\text { CHS en TS } \\
(\%)\end{array}$ \\
\hline 0 & 100,0 & 100,0 & 100,0 \\
26 & 98,5 & 64,2 & 60,1 \\
35 & 89,2 & 57,1 & 41,9 \\
54 & 85,8 & 41,0 & 24,3 \\
82 & 83,5 & 29,9 & 19,0 \\
117 & 90,5 & 91,6 & 93,3 \\
\hline
\end{tabular}

Los valores corresponden a las medias de contenido hídrico del sustrato (CHS) de cada uno de los tratamientos al momento de la evaluación. $\mathrm{TC}=$ Tratamiento Control; $\mathrm{TM}=$ Tratamiento Moderado; $\mathrm{TS}=$ Tratamiento Severo.

Values correspond to the soil water content (CHS) of each treatment at the evaluation period. $\mathrm{TC}=$ Control treatment; $\mathrm{TM}=$ Moderate treatment; $\mathrm{TS}=$ Severe treatment. timación de los parámetros se hizo utilizando una transformación (tipo II) que relaciona el inverso del potencial hídrico total con el respectivo contenido hídrico relativo de la muestra [2]:

$$
\frac{1}{\psi_{t}}=a \times C H R+b
$$

Donde $\Psi_{t}=$ potencial hídrico total y se obtiene de la suma del potencial osmótico $(\pi)$ y el potencial de presión $\left(\Psi_{p}\right)$; $a$ y $b=$ coeficientes de regresión, y $C H R=$ contenido hídrico relativo de la muestra al momento de la medición.

Evaluación de variables de la fluorescencia de las clorofilas e intercambio gaseoso. Junto con las mediciones de variables hídricas, se evaluó el comportamiento de la fluorescencia de las clorofilas utilizando un fluorímetro modulado portátil (Mini-PAM Photosynthesis Yield Analyser. Walz, Effeltrich, Alemania). Un pulso de luz de fotones de aproximadamente $0,5 \mu \mathrm{mol} \mathrm{m} \mathrm{m}^{-2} \mathrm{~s}^{-1}$ fue configurado a una frecuencia de $600 \mathrm{~Hz}$ para determinar, a pre-alba, la señal de fluorescencia mínima $\left(\mathrm{F}_{0}\right)$. Para obtener la señal de fluorescencia máxima $\left(\mathrm{F}_{\mathrm{m}}\right)$ se aplicó un pulso de saturación de fotones de aproximadamente $6.000 \mu \mathrm{mol} \mathrm{m}^{-2} \mathrm{~s}^{-1}$ durante $0,8 \mathrm{~s}$. El rendimiento cuántico máximo del fotosistema II (FSII) a pre-alba fue calculado como $F_{v} / F_{m}=\left(F_{m}-F_{0}\right) / F_{m}$. Al mediodía, la fluorescencia estable adaptada a la luz $\left(\mathrm{F}_{t}\right)$ y la fluorescencia máxima adaptada a la luz $\left(\mathrm{F}_{\mathrm{m}}^{\prime}\right)$, fueron determinadas en las mismas hojas utilizadas en la medición de pre-alba, aplicando el mismo procedimiento. La eficiencia fotoquímica del FSII $\left(\Phi_{\mathrm{PSII}}\right)$ fue determinada como $\Phi_{\mathrm{PSII}}=\left(\mathrm{F}_{\mathrm{m}}^{\prime}-\mathrm{F}_{\mathrm{t}}\right) / \mathrm{F}_{\mathrm{m}}^{\prime}$. La hoja utilizada durante la medición del mediodía fue adaptada a la oscuridad durante un periodo de 20-25 minutos, lo cual permitió calcular el rendimiento cuántico máximo del FSII al mediodía como: $\mathrm{F}_{\mathrm{v}}^{\prime} / \mathrm{F}_{\mathrm{m}}^{\prime}=\left(\mathrm{F}_{\mathrm{m}}^{\prime}-\mathrm{F}_{0}\right) / \mathrm{F}_{\mathrm{m}}^{\prime}$.

Paralelamente, en la mañana (de 10:00 a 11:30 horas), se hicieron evaluaciones puntuales de los parámetros de intercambio gaseoso utilizando un aparato portátil de fotosíntesis $($ IRGA $)\left(\mathrm{ADC}-\mathrm{LCi}, \mathrm{UK}-\mathrm{CO}_{2}\right.$ ambiental $\left.=380 \mathrm{ppm}\right)$. Se obtuvo la fotosíntesis neta (An), conductancia estomática (gs), transpiración (E) y concentración interna de $\mathrm{CO}_{2}$ (Ci). Las evaluaciones de fluorescencia e intercambio gaseoso se hicieron en una muestra de cinco individuos por tratamiento y en momentos representativos del gradiente de CHS: día 0 (TC: $100 \%$, TM: $100 \%$, TS: $100 \%$ ); día 35 (TC: $89 \%$, TM: $57 \%$, TS: $42 \%$ ) y día 82 (TC: $84 \%$, TM: $30 \%$, TS: $19 \%$ ).

Evaluación de variables de crecimiento y distribución de biomasa. Se efectuó un seguimiento de las variables de crecimiento durante todo el periodo de estudio. $\mathrm{Al}$ inicio del ensayo se seleccionaron 20 plantas por tratamiento, que fueron marcadas en la sección inferior del tallo principal con el objeto de determinar la evolución del diámetro a la altura 
del cuello (DAC) y altura total (L). Para las mediciones de altura se utilizó el vástago principal de cada planta, registrando la distancia desde la marca hasta el ápice del vástago con una cinta métrica de precisión al milímetro. En el caso del DAC, las mediciones se hicieron con un pie de metro con precisión al milímetro. El monitoreo de estas variables se hizo posterior a la evaluación de variables hídricas.

Terminado el periodo de restricción hídrica, se determinó en diez individuos de cada tratamiento la biomasa aérea y radicular. De cada planta seleccionada se separó el sistema de raíces de la parte aérea, el que a su vez se dividió en hojas y material leñoso. Estas muestras se secaron a $65^{\circ} \mathrm{C}$ en una estufa de aire forzado hasta alcanzar un peso estable, obteniendo la biomasa total y por componente. Estos datos se compararon con la biomasa inicial obtenida de una muestra de diez plantas del total de plantas utilizadas en el ensayo. Con los antecedentes obtenidos se determinó la relación entre la biomasa aérea y biomasa de raíces de las plantas según tratamiento.

Análisis estadístico. Las diferencias en los parámetros derivados del análisis de las curvas presión-volumen, variables de intercambio gaseoso, fluorescencia de las clorofilas, potencial hídrico y biomasa, se evaluaron con un análisis de varianza, para un diseño completamente al azar de tres tratamientos. El crecimiento fue analizado mediante análisis de varianza de medidas repetidas. Para determinar la significancia de las diferencias entre los valores medios de cada tratamiento, se utilizó la prueba de Tukey $(P \leq 0,05)$. Para el análisis de las variables de crecimiento se utilizó el DAC inicial o la altura inicial como covariables.

\section{RESULTADOS}

Estado hídrico de los individuos durante el periodo de estudio. Las plantas del tratamiento control mostraron valores de contenido hídrico del sustrato superior al $70 \%$, lo que permitió el mantenimiento de valores de potencial hídrico a pre-alba y contenidos hídricos relativos a pre-alba en torno a $-0,4 \mathrm{MPa}$ y $96 \%$, respectivamente (figura 1). A partir del día 12, los tratamientos de restricción hídrica moderada y severa experimentaron un descenso en el valor promedio del contenido hídrico del sustrato, observándose diferencias estadísticamente significativas en $\Psi_{\mathrm{a}}$ y $\mathrm{CHR}_{\mathrm{a}}$ solo a partir del día 54, momento en el cual el contenido hídrico del sustrato fue de $42 \%$ en las plantas con tratamiento de restricción hídrica moderada, y $26 \%$ en las plantas con tratamiento de restricción hídrica severa. El periodo de mayores limitaciones en las plantas con tratamientos de restricción hídrica se presentó entre los días $55 \mathrm{y}$ 82 , cuando las plantas presentaron valores promedio de contenido hídrico del sustrato de $29,9 \%$ TM, y $19 \%$ en TS. Esta condición, de menor disponibilidad hídrica en el sustrato, provocó una baja importante en el potencial hídrico, especialmente en el tratamiento de restricción hídrica severa $\left(-4,3 \mathrm{MPa}\right.$ en $\Psi_{\mathrm{a}}$ y $84,8 \%$ en $\left.\mathrm{CHR}_{\mathrm{a}}\right)$. Posterior al día 82, los dos tratamientos con restricción hídrica fueron regados, entrando a una fase de rehidratación (lo que se vio reflejado en un aumento del valor promedio del contenido hídrico del sustrato), alcanzando rápidamente valores de $\Psi_{\text {a }}$ y $\mathrm{CHR}_{\mathrm{a}}$ similares a los observados en las plantas que recibieron riego permanente (figura 1).
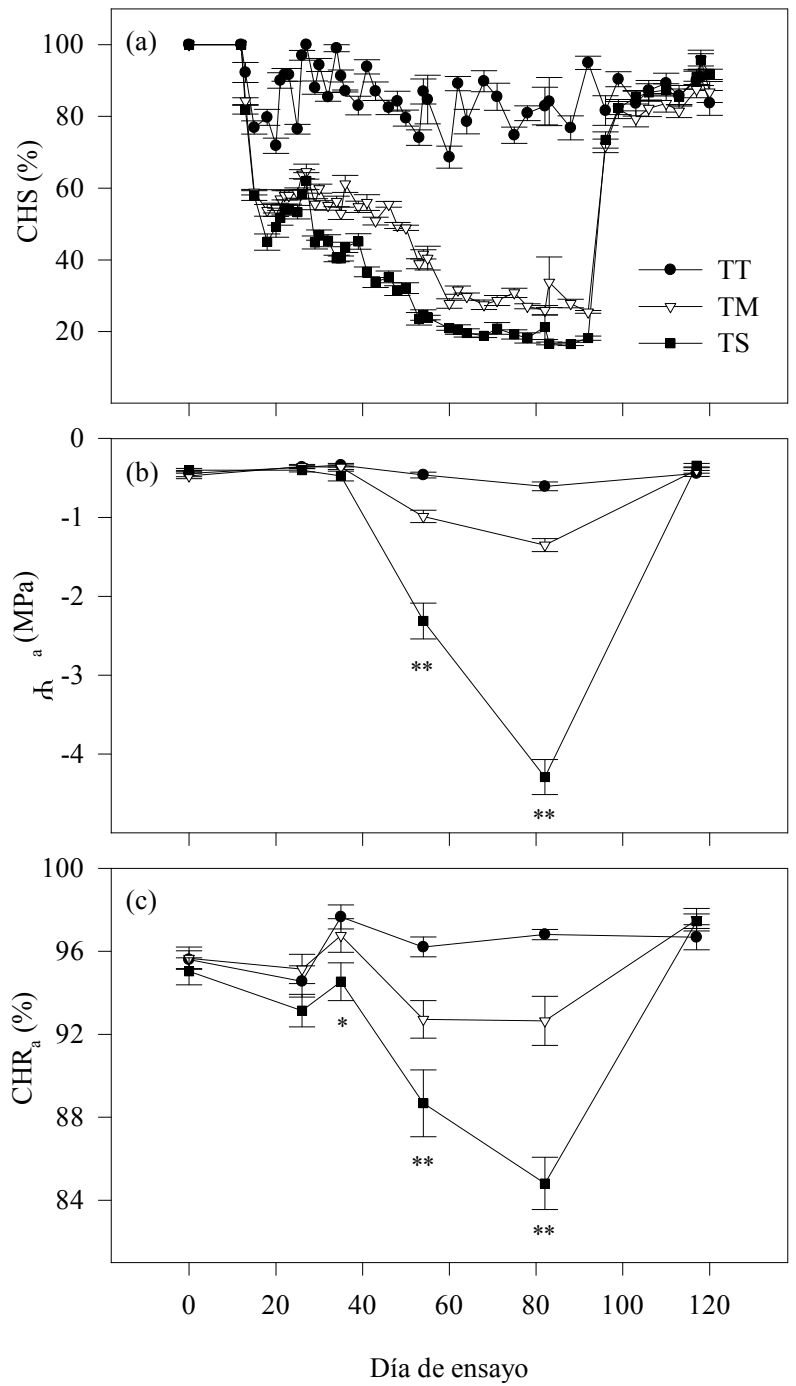

Figura 1. Comportamiento de las variables hídricas durante el periodo de estudio. a) contenido hídrico del sustrato $=$ CHS, b) potencial hídrico foliar a pre-alba $=\Psi_{a}$, c) contenido hídrico relativo foliar a pre-alba $=\mathrm{CHR}_{\mathrm{a}}$. TT: Tratamiento Testigo o control, $\mathrm{TM}=$ Tratamiento Moderado; $\mathrm{TS}=$ Tratamiento Severo. Valores corresponden a medias \pm error estándar $(\mathrm{n}=8-10)$. La significancia del tratamiento de restricción hídrica sobre las variables $\Psi_{\text {a }}$ y $\mathrm{CHR}_{\mathrm{a}}$ se indica en asteriscos $(* P \leq 0,05, * * P \leq 0,01)$.

Water variables during the evaluation period. a) soil water content (CHS), b) predawn leaf relative water content $\left(\mathrm{CHR}_{\mathrm{a}} \mathrm{TC}=\mathrm{Con}-\right.$ trol treatment; $\mathrm{TM}=$ Moderate treatment; $\mathrm{TS}=$ Severe treatment. Values correspond to means $\pm \mathrm{SE}(\mathrm{n}=8-10)$. The significance of the water restriction treatment on the variables $\Psi \mathrm{a}$ and CHRa is indicated by asterisks (* $P \leq 0.05$, ** $P \leq 0.01$ ). 
Parámetros derivados de las curvas presión-volumen. El potencial osmótico a cero turgor y a turgor máximo fueron afectados por la restricción hídrica, siendo significativamente más negativos a los valores observados después de 82 días de tratamiento, momento en que ambas condiciones de restricción hídrica (TM y TS) alcanzaron los mayores niveles de estrés (cuadro 2). Valores más negativos de $\pi_{0} \mathrm{y} \pi_{100}$, con un $\mathrm{CHR}_{0}$ sobre $80 \%$, es un indicador de ajuste osmótico.

Intercambio gaseoso y fluorescencia de las clorofilas. Mientras el contenido hídrico del sustrato se mantuvo sobre el $40 \%$, no se observaron diferencias entre los tratamientos en los parámetros de intercambio gaseoso (figura 2). Sin embargo, cuando el CHS se situó por debajo del $30 \%$ (día 82), se observó una disminución significativa en los valores promedio de fotosíntesis neta, conductancia estomática y transpiración, en los tratamientos con restricción hídrica moderada y severa.
Respecto a la eficiencia fotoquímica del FSII, esta no presentó diferencias entre los tratamientos en todas las evaluaciones. Sin embargo, se observó en todos los tratamientos una tendencia al descenso en el valor de $\Phi_{\mathrm{PSII}}$ a medida que transcurre la estación, indicando una disminución en la proporción de luz absorbida que está siendo utilizada en la fotosíntesis. El rendimiento cuántico máximo del fotosistema II disminuyó al mediodía y aumentó a prealba, observándose diferencias significativas en el período de mayor estrés (figura 3 ).

Crecimiento y distribución de la biomasa. El crecimiento en diámetro a la altura del cuello fue afectado directamente por la reducción de fotosíntesis neta en función del aporte hídrico de cada tratamiento (figura 4). Durante la evaluación realizada el día $68(\mathrm{CHS} \mathrm{TC}=89,8 \%$, CHS TM $=27,5 \%$, CHS TS $=18,9 \%$ ), las plantas del tratamiento con restricción hídrica moderada presentaban un valor promedio de potencial hídrico a pre-alba por debajo de -1,1 MPa, lo que

Cuadro 2. Parámetros hídricos de plantas de Lithraea caustica derivados de las curvas presión-volumen, según tratamiento y día de evaluación.

Water relation parameters of Lithraea caustica plants derived from the pressure-volume curves, according to treatment and day.

\begin{tabular}{cccccccc}
\hline Día & CHS (\%) & Tratamiento & $\mathrm{CHR}_{0}(\%)$ & $\pi_{100}(\mathrm{MPa})$ & $\pi_{0}(\mathrm{MPa})$ & $\Psi_{\mathrm{P} 100}(\mathrm{MPa})$ & $\varepsilon$ \\
\hline 0 & 100,0 & TC & $84,9 \pm 0,0 \mathrm{Aa}$ & $-2,5 \pm 0,1 \mathrm{Ac}$ & $-3,2 \pm 0,1 \mathrm{Ab}$ & $2,3 \pm 0,1 \mathrm{Ac}$ & $18,0 \pm 1,4 \mathrm{Aa}$ \\
& 100,0 & TM & $87,2 \pm 0,0 \mathrm{Aa}$ & $-2,3 \pm 0,2 \mathrm{Aab}$ & $-3,1 \pm 0,2 \mathrm{Abc}$ & $2,1 \pm 0,2 \mathrm{Aab}$ & $17,1 \pm 1,2 \mathrm{Aab}$ \\
& 100,0 & TS & $85,4 \pm 0,0 \mathrm{Aa}$ & $-2,4 \pm 0,1 \mathrm{Ab}$ & $-3,1 \pm 0,1 \mathrm{Ab}$ & $2,2 \pm 0,1 \mathrm{Ac}$ & $16,9 \pm 1,0 \mathrm{Aa}$ \\
35 & 91,7 & TC & $89,7 \pm 0,0 \mathrm{Ba}$ & $-1,8 \pm 0,1 \mathrm{Aa}$ & $-2,3 \pm 0,1 \mathrm{Aa}$ & $1,4 \pm 0,1 \mathrm{Aa}$ & $15,1 \pm 1,3 \mathrm{Aa}$ \\
& 60,2 & TM & $87,7 \pm 0,0 \mathrm{ABa}$ & $-2,0 \pm 0,1 \mathrm{Aa}$ & $-2,5 \pm 0,1 \mathrm{Aa}$ & $1,5 \pm 0,0 \mathrm{Aa}$ & $14,0 \pm 1,3 \mathrm{Aab}$ \\
54 & 42,0 & TS & $86,1 \pm 0,0 \mathrm{Aa}$ & $-1,9 \pm 0,1 \mathrm{Aa}$ & $-2,5 \pm 0,1 \mathrm{Aa}$ & $1,5 \pm 0,1 \mathrm{Aa}$ & $13,0 \pm 1,3 \mathrm{Aa}$ \\
& 85,9 & TC & $88,7 \pm 0,0 \mathrm{Ba}$ & $-2,0 \pm 0,1 \mathrm{Aab}$ & $-2,5 \pm 0,1 \mathrm{Aa}$ & $1,6 \pm 0,1 \mathrm{Aab}$ & $15,5 \pm 0,7 \mathrm{Aa}$ \\
& 41,6 & TM & $81,9 \pm 0,0 \mathrm{Aa}$ & $-2,3 \pm 0,1 \mathrm{ABab}$ & $-3,0 \pm 0,1 \mathrm{Bab}$ & $1,9 \pm 0,1 \mathrm{Aab}$ & $13,0 \pm 1,4 \mathrm{Aa}$ \\
& 25,6 & TS & $82,7 \pm 0,0 \mathrm{ABa}$ & $-2,4 \pm 0,1 \mathrm{Bb}$ & $-3,1 \pm 0,1 \mathrm{Bb}$ & $1,9 \pm 0,1 \mathrm{Aab}$ & $13,3 \pm 2,0 \mathrm{Aa}$ \\
& 83,5 & TC & $87,7 \pm 0,0 \mathrm{Aa}$ & $-2,5 \pm 0,2 \mathrm{Ac}$ & $-3,1 \pm 0,2 \mathrm{Ab}$ & $2,0 \pm 0,2 \mathrm{Abc}$ & $18,3 \pm 0,9 \mathrm{Aa}$ \\
& 29,9 & TM & $82,5 \pm 0,0 \mathrm{Aa}$ & $-2,8 \pm 0,0 \mathrm{Ac}$ & $-3,6 \pm 0,1 \mathrm{Bc}$ & $2,2 \pm 0,1 \mathrm{Ab}$ & $16,1 \pm 1,8 \mathrm{Aab}$ \\
& 117 & TS & $86,1 \pm 0,0 \mathrm{Aa}$ & $-2,9 \pm 0,1 \mathrm{Ac}$ & $-3,6 \pm 0,1 \mathrm{Bc}$ & $2,0 \pm 0,1 \mathrm{Ab}$ & $15,5 \pm 0,7 \mathrm{Aa}$ \\
& 91,3 & TC & $85,9 \pm 0,0 \mathrm{Aa}$ & $-2,3 \pm 0,0 \mathrm{Abc}$ & $-3,3 \pm 0,1 \mathrm{Ab}$ & $2,0 \pm 0,1 \mathrm{Abc}$ & $17,1 \pm 0,8 \mathrm{Aa}$ \\
& 90,0 & TM & $86,0 \pm 0,0 \mathrm{Aa}$ & $-2,5 \pm 0,1 \mathrm{Abc}$ & $-3,4 \pm 0,1 \mathrm{Abc}$ & $2,3 \pm 0,1 \mathrm{Ab}$ & $18,9 \pm 1,1 \mathrm{Ab}$ \\
& 93,5 & TS & $85,7 \pm 0,0 \mathrm{Aa}$ & $-2,7 \pm 0,1 \mathrm{Abc}$ & $-3,5 \pm 0,1 \mathrm{Ac}$ & $2,1 \pm 0,1 \mathrm{Ab}$ & $17,2 \pm 1,2 \mathrm{Aa}$ \\
\hline
\end{tabular}

Los valores corresponden a medias \pm error estándar $(\mathrm{n}=6)$. Contenido hídrico relativo a cero turgor $\left(\mathrm{CHR}_{0}\right)$; Potencial osmótico a turgor máximo $\left(\pi_{100}\right)$; Potencial osmótico a cero turgor $\left(\pi_{0}\right)$; Potencial de presión a turgor máximo $\left(\Psi_{\mathrm{P} 100}\right)$; Módulo de elasticidad de la pared celular $(\varepsilon)$. TC= Tratamiento Control; $\mathrm{TM}=$ Tratamiento Moderado; $\mathrm{TS}=$ Tratamiento Severo. Letras mayúsculas distintas indican diferencias significativas entre tratamientos, para cada día de evaluación. Letras minúsculas distintas indican diferencias significativas dentro del tratamiento (Tukey, $P \leq 0,05$ ).

Values correspond to means \pm standard error $(\mathrm{n}=6)$. Relative water content relative to turgor loss point $\left(\mathrm{CHR}_{0}\right)$; Osmotic potential at full turgor $\left(\pi_{100}\right)$; Osmotic potential at turgor loss point $\left(\pi_{0}\right)$; Pressure potential at full turgor $\left(\Psi_{100}\right)$; bulk elastic module $(\varepsilon)$. TC $=$ Control; TM= Moderate treatment; TS= Severe treatment. Differing capital letters indicate significant differences among treatments in each day. Differing lowercase letters indicate significant differences within treatment (Tukey, $P \leq 0.05$ ). 

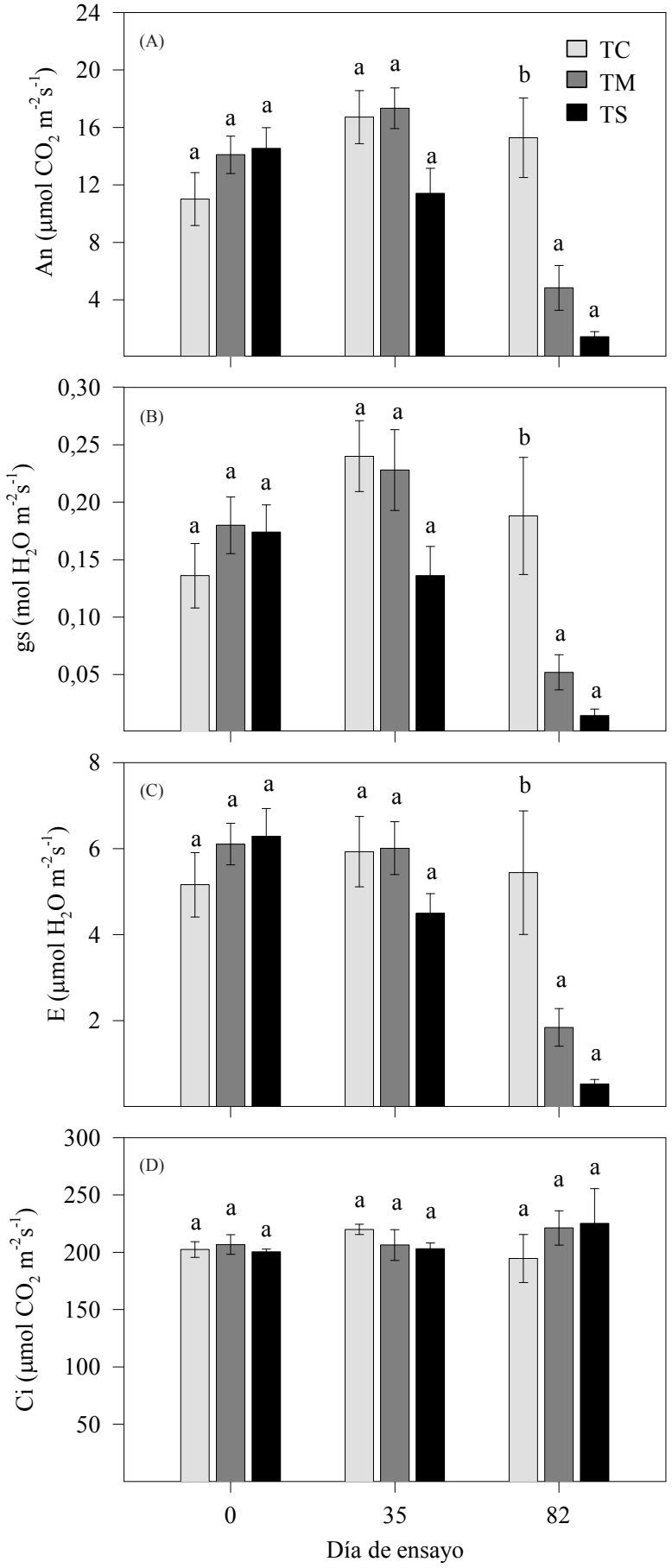

Figura 2. Variables de intercambio gaseoso de plantas de $\mathrm{Li}$ thraea caustica según tratamiento y día de evaluación. A) Fotosíntesis neta $=\mathrm{A}_{\mathrm{n}}, \mathrm{B}$ ) conductancia estomática $=\mathrm{g}_{\mathrm{s}}, \mathrm{C}$ ) transpiración $=\mathrm{E}, \mathrm{D})$ concentración interna de $\mathrm{CO}_{2}=\mathrm{C}_{\mathrm{i}}$. Valores corresponden a medias \pm error estándar $(n=5)$. Letras distintas indican diferencias significativas entre los tratamientos (Tukey, $P \leq 0,05$ ).

Gas exchange rates of Lithraea caustica plants as a function of treatment and day. A) Net photosynthesis $\left.\left(A_{n}\right), B\right)$ Stomatal conductance $=\mathrm{g}_{\mathrm{s}}, \mathrm{C}$ ) Transpiration $\left.=\mathrm{E}, \mathrm{D}\right)$ Intercellular $\mathrm{CO}_{2}=\mathrm{C}_{\mathrm{i}}$ concentration. Values are mean $\pm S E(n=5)$. Different letters indicate significant differences among treatments (Tukey, $P \leq 0.05$ ). significó una reducción en la tasa de crecimiento en diámetro con respecto a la tasa que presentó el tratamiento con riego permanente (TC). Las plantas del tratamiento con restricción hídrica severa presentaron, durante el mismo periodo, un valor promedio de $\Psi_{\text {a }}$ por debajo de -2,3 $\mathrm{MPa}$, lo que resultó en una detención del crecimiento en diámetro producto de la restricción hídrica. Esta condición se
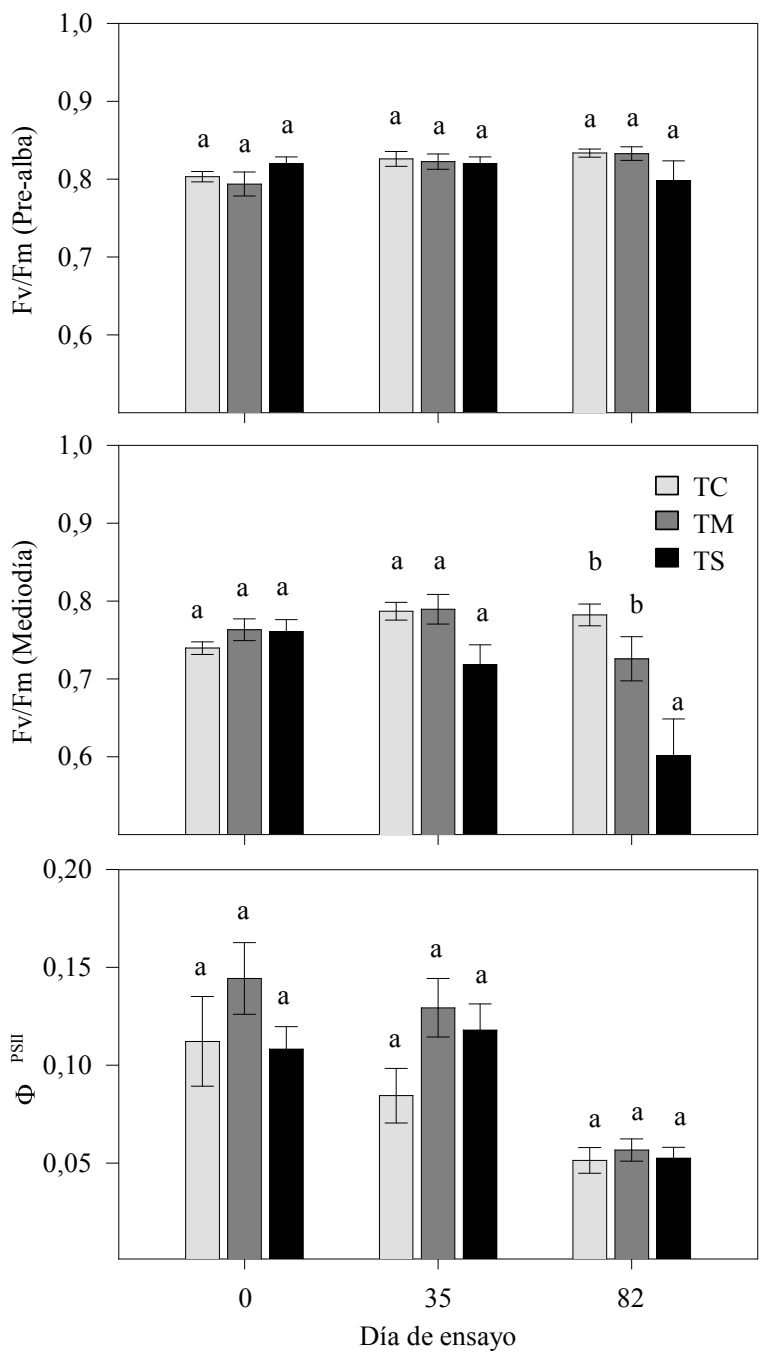

Figura 3. Variables de la fluorescencia de las clorofilas de plantas de Lithraea caustica según tratamiento y día de evaluación. $\mathrm{F}_{\mathrm{v}} / \mathrm{F}_{\mathrm{m}}($ Pre-alba $)=$ Rendimiento cuántico máximo del fotosistema II pre-alba, $\mathrm{F}_{\mathrm{v}} / \mathrm{F}_{\mathrm{m}}$ (mediodía) $=$ Rendimiento cuántico máximo del fotosistema II al mediodía, $\Phi_{\mathrm{PSII}}=$ Eficiencia fotoquímica del PSII. Valores corresponden a medias \pm error estándar $(n=5)$. Letras distintas indican diferencias significativas entre tratamientos (Tukey, $P \leq 0,05$ ).

Chlorophyll fluorescence of Lithraea caustica plants as a function of treatment and day. $\mathrm{F}_{\gamma} / \mathrm{F}_{\mathrm{m}}$ (Predawn) $=$ maximum quantum yield of photosystem II at predawn, $\mathrm{F}_{\mathrm{v}} / \mathrm{F}_{\mathrm{m}}$ (Midday) = maximum quantum yield of photosystem II at midday, $\Phi_{\mathrm{PSII}}=$ Photochemical efficiency of PSII Values are mean $\pm \mathrm{SE}(\mathrm{n}=5)$. Different letters indicate significant differences among treatments (Tukey, $P \leq 0.05$ ). 
mantuvo hasta la evaluación del día 89 (CHS TC $=76,8 \%$, CHS TM $=27,8 \%$, CHS TS $=16,5 \%$, periodo en el cual ambos tratamientos de restricción hídrica alcanzaron los niveles más altos de estrés. Por otra parte, el crecimiento en altura mostró un comportamiento errático, sin observar una tendencia clara entre los tratamientos (figura 4).

Las plantas incrementaron significativamente su valor de biomasa respecto al valor obtenido al inicio del ensayo en todos sus componentes (hojas, ramas y raíces), en todos los tratamientos (cuadro 3). No se encontraron diferencias estadísticas en el valor promedio de biomasa total en plantas con restricción hídrica moderada o severa y sin restricción hídrica (cuadro 3).
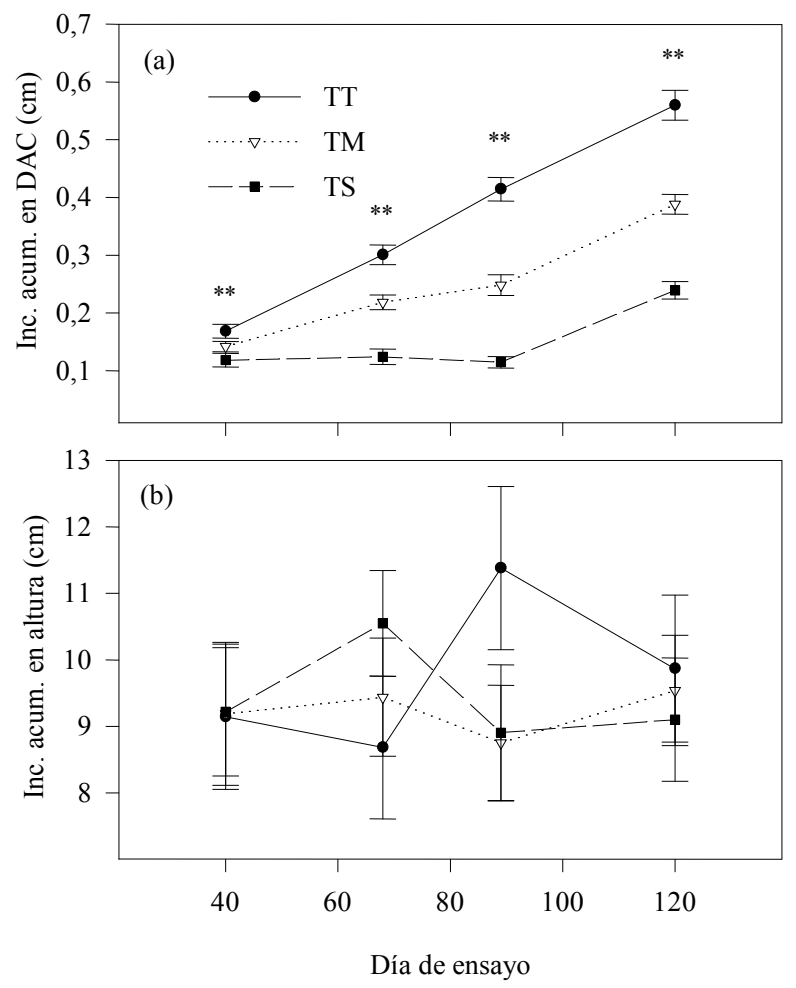

Figura 4. Incremento acumulado de las variables de crecimiento en plantas de Lithraea caustica durante el periodo de estudio. a) Incremento acumulado en DAC, b) Incremento acumulado en altura. Valores corresponden a medias \pm error estándar $(n=20)$. TT: Tratamiento Testigo o control, TM= Tratamiento Moderado; $\mathrm{TS}=$ Tratamiento Severo. ${ }^{*}$ *indica la existencia de diferencia significativa entre los tres tratamientos de riego al $P \leq 0,01$.

Accumulated increase of growth variables in Lithraea caustica plants during the evaluation period. a) Accumulated increase in basal diameter (DAC), b) Accumulated increase in height. Values are mean $\pm \mathrm{SE}(\mathrm{n}=20) . \mathrm{TC}=$ Control treatment; $\mathrm{TM}=$ Moderate treatment; $\mathrm{TS}=$ Severe treatment. $* *$ indicates the existence of a significant difference among water restriction treatments at $P \leq 0.01$.

\section{DISCUSIÓN}

Las respuestas de las variables hídricas en función del contenido hídrico del sustrato concuerdan con estudios efectuados en otras especies mediterráneas (Galmés et al. 2007, Gallé et al. 2007). Del mismo modo, Donoso et al. (2011) reportan un comportamiento similar en las variables $\Psi_{\mathrm{a}}$ y $\mathrm{CHR}_{\mathrm{a}}$ en plantas de Quillaja saponaria y Cryptocarya alba (peumo) sometidas a restricción hídrica, en condiciones ambientales similares. En estas especies, los valores de contenido hídrico del sustrato a los cuales se presentan los máximos niveles de restricción hídrica, sin mortalidad de plantas, es de un $40 \%$ en el caso de Q. saponaria, y $43 \%$ en el caso de C. alba, valores que difieren notablemente con el obtenido para $L$. caustica en este estudio (19\%), sugiriendo una mayor habilidad de L. caustica para tolerar condiciones de restricción hídrica. No obstante, Acacia caven, presentaría mayor tolerancia que $L$. caustica a períodos de estrés, ya que, en un ensayo similar, a los 61 días de restricción hídrica y niveles de $9 \%$ y $10 \%$ de CHS, se registran los menores niveles de $\Psi_{\text {a }}$ y CHR, respectivamente (Donoso et al. 2015)

Por otra parte, bajo condiciones de restricción hídrica L. caustica desarrolló el mecanismo de ajuste osmótico. Este es un proceso mediado por la acumulación activa de solutos en las células vegetales, lo cual hace que el valor de $\pi_{0}$ y $\pi_{100}$ sea más negativo, presentando un alto turgor celular, manteniendo el contenido hídrico relativo foliar sobre $80 \%$. Esta estrategia permite a L. caustica tolerar los bajos contenidos hídricos de sustrato observados durante la fase de mayor restricción hídrica. Del mismo modo, Donoso et al. (2011) reportan, bajo condiciones de ensayo similares, que $C$. alba (especie siempreverde) realiza ajuste osmótico, donde el valor de $\pi_{0}$ se hace más negativo bajo condiciones de restricción hídrica, aunque sin presentar un cambio significativo en el valor de $\pi_{100}$.

Los valores de contenido hídrico relativo a cero turgor que se observaron durante el transcurso del ensayo, no mostraron un cambio significativo a lo largo del periodo de estudio, posiblemente a causa de la baja elasticidad que presentaron las paredes celulares producto de los altos valores obtenidos en el módulo de elasticidad de la pared celular. Sin embargo, estos se mantuvieron dentro de los rangos informados en otros estudios realizados bajo condiciones de estrés con especies mediterráneas (Corcuera et al. 2002).

Comparando el comportamiento que presentó el potencial hídrico a pre-alba durante el ensayo con el punto de pérdida de turgencia $(-3,6 \mathrm{MPa})$, obtenido mediante el análisis de las curvas presión-volumen, se infiere que el tratamiento de restricción hídrica moderada mantuvo durante todo el ensayo valores de $\Psi_{\mathrm{a}}$ sobre el punto de pérdida de turgencia. Por otra parte, el tratamiento con restricción hídrica severa presentó valores promedio de $\Psi_{\text {a }}$ bajo -3,6 $\mathrm{MPa}$, con efectos negativos sobre el intercambio gaseoso $\mathrm{y}$, por lo tanto, una reducción en el crecimiento diametral (DAC). 
Cuadro 3. Componentes de biomasa en plantas de Lithraea caustica según periodo de evaluación y tratamiento aplicado.

Biomass distribution of Lithraea caustica plants as a function of evaluation period and treatments.

\begin{tabular}{|c|c|c|c|c|}
\hline \multirow{2}{*}{ Componente de biomasa } & \multirow{2}{*}{ Inicio del ensayo } & \multicolumn{3}{|c|}{ Fin de la restricción hídrica (día 90) } \\
\hline & & $\mathrm{TC}$ & $\mathrm{TM}$ & TS \\
\hline Hojas (g) & $\begin{array}{c}10,6 \pm 1,4 \mathrm{a} \\
(43,5 \%)\end{array}$ & $\begin{array}{c}22,7 \pm 1,5 \mathrm{Bb} \\
(35,8 \%)\end{array}$ & $\begin{array}{c}20,5 \pm 1,4 \mathrm{Bb} \\
(34,7 \%)\end{array}$ & $\begin{array}{c}15,4 \pm 0,9 \mathrm{Ab} \\
(27,6 \%)\end{array}$ \\
\hline Tallos $(\mathrm{g})$ & $\begin{array}{c}6,0 \pm 0,9 a \\
(24,5 \%)\end{array}$ & $\begin{array}{c}21,1 \pm 2,3 \mathrm{Ab} \\
(32,2 \%)\end{array}$ & $\begin{array}{c}20,9 \pm 2,0 \mathrm{Ab} \\
(34,6 \%)\end{array}$ & $\begin{array}{c}20,0 \pm 1,1 \mathrm{Ab} \\
(35,9 \%)\end{array}$ \\
\hline Raíz (g) & $\begin{array}{c}7,9 \pm 1,2 \mathrm{a} \\
(32,0 \%)\end{array}$ & $\begin{array}{c}20,6 \pm 1,8 \mathrm{Ab} \\
(32,0 \%)\end{array}$ & $\begin{array}{c}17,9 \pm 1,1 \mathrm{Ab} \\
(30,6 \%)\end{array}$ & $\begin{array}{c}20,9 \pm 2,1 \mathrm{Ab} \\
(36,5 \%)\end{array}$ \\
\hline Total (g) & $24,4 \pm 3,3$ a & $64,5 \pm 5,2 \mathrm{Ab}$ & $59,4 \pm 3,8 \mathrm{Ab}$ & $56,3 \pm 3,3 \mathrm{Ab}$ \\
\hline $\mathrm{BA} / \mathrm{BR}$ & $2,3 \pm 0,2 \mathrm{a}$ & $2,2 \pm 0,1 \mathrm{ABa}$ & $2,3 \pm 0,2 \mathrm{Ba}$ & $1,8 \pm 0,1 \mathrm{Aa}$ \\
\hline
\end{tabular}

Los valores corresponden a medias \pm error estándar $(n=10)$. Entre paréntesis se indica la proporción del componente respecto del total de biomasa. Letras mayúsculas distintas indican diferencias significativas entre tratamientos, para la evaluación del día 90. Letras minúsculas distintas indican diferencias significativas entre la biomasa al inicio y al final del periodo de restricción hídrica (Tukey, $P \leq 0,05)$. TC $=$ Tratamiento Control; TM= Tratamiento Moderado; TS= Tratamiento Severo, BA/BR = Biomasa Aérea $/$ Biomasa Radical.

Data in parenthesis correspond to the percentage of total dry matter. Different capital letters indicate significant differences among treatments for the evaluation of day 90. Different lower case letters indicate significant differences between biomass distribution at the beginning and end of the water restriction period (Tukey, $P \leq 0.05$ ). $\mathrm{TC}=$ Control; $\mathrm{TM}=$ Moderate treatment; $\mathrm{TS}=$ Severe treatment. $\mathrm{BA} / \mathrm{BR}=$ Above ground biomass $/$ root biomass.

Respecto al intercambio gaseoso y fluorescencia de las clorofilas, $L$. caustica tuvo un comportamiento similar al de $Q$. saponaria y $C$. alba, que muestran disminuciones significativas en sus valores de fotosíntesis y conductancia estomática bajo condiciones de restricción hídrica severa (Donoso et al. 2011). Según Grassi y Magnani (2005), cuando la conductancia estomática máxima cae por debajo de $0,1 \mathrm{~mol} \mathrm{H}_{2} \mathrm{O} \mathrm{m}^{-2} \mathrm{~s}^{-1}$, se produce un mal funcionamiento general en el metabolismo de la planta, situación que ocurrió en ambos tratamientos con restricción hídrica, e indicaría que a partir de contenidos hídricos del sustrato por debajo del $30 \%$, L. caustica comenzaría a manifestar claros síntomas de estrés por déficit de agua, provocando una respuesta para mejorar la eficiencia en el uso de este recurso, principalmente a través del cierre estomático (Peña-Rojas et al. 2004, Fleck et al. 2010).

Sin embargo, pese a la fuerte restricción hídrica a la que fueron sometidas las plantas del tratamiento con restricción hídrica severa (aproximadamente 19\% CHS), los valores de conductancia estomática y fotosíntesis neta no llegaron a cero, como observan Donoso et al. (2011) en C. alba. Esto indicaría una mayor habilidad de L. caustica en tolerar condiciones de restricción hídrica, posiblemente debido a un mayor control de la deshidratación a través del ajuste osmótico. Un comportamiento similar observó Fotelli et al. (2000) en plantas de la especie Quercus macrolepis Kotschy, las que aparentemente tienen la habilidad de superar el estrés hídrico a través de mecanismos de tolerancia a la deshidratación, lo que se ve reflejado en una alta conductancia estomática aún a bajos potenciales hídricos a pre-alba. La misma tendencia es registrada para $A$. caven
(Donoso et al. 2015). Los resultados obtenidos en las variables de intercambio gaseoso, sugieren que $L$. caustica sería capaz de sobrellevar el periodo de restricción hídrica por medio del ajuste osmótico, pero manteniendo la fotosíntesis y el intercambio gaseoso, a una tasa reducida.

En condiciones normales, la mayoría de las plantas superiores posee un rendimiento cuántico máximo del fotosistema II $\left(\mathrm{F}_{\mathrm{v}} / \mathrm{F}_{\mathrm{m}}\right)$ a pre-alba óptimo cercano a 0,83 , mientras que al mediodía $\mathrm{F}_{\mathrm{v}} / \mathrm{F}_{\mathrm{m}}$ oscila entre 0,7 y 0,8 , valores típicos de plantas sanas (Krause y Weis 1991, Maxwell y Johnson 2000, Peña-Rojas et al. 2004). Para L. caustica, el rendimiento cuántico máximo del fotosistema II a prealba se mantuvo estable durante las evaluaciones de los días 0,35 y 82 , no mostrando diferencias estadísticas entre los tratamientos, con valores promedios de 0,8 . Sin embargo, cuando la restricción hídrica fue más fuerte (día 82), se produjo una disminución significativa en los valores de $\mathrm{F}_{\mathrm{v}} / \mathrm{F}_{\mathrm{m}}$ al mediodía, en el tratamiento con restricción hídrica severa.

Se ha observado en especies arbóreas mediterráneas que frente a estrés hídrico ocurre una reducción significativa de $\mathrm{F}_{\mathrm{v}} / \mathrm{F}_{\mathrm{m}}$ a pre-alba bajo los rangos establecidos, que implica que los fotosistemas son afectados durante el día y no logran recuperarse durante la noche (Peña-Rojas et al. 2004, Fleck et al. 2010). Para el caso de L. caustica, existe una disminución de $\mathrm{F}_{\mathrm{v}} / \mathrm{F}_{\mathrm{m}}$ al mediodía, pero una posterior recuperación durante la noche, lo que se refleja en los valores de $\mathrm{F}_{\mathrm{v}} / \mathrm{F}_{\mathrm{m}}$ a pre-alba. Esto sugiere que L. caustica es capaz de reparar los daños provocados durante el periodo de fotoinhibición observado a mediodía (Fleck et al. 2010).

La limitación del crecimiento y acumulación de biomasa debido al estrés hídrico es causada principalmente por 
la reducción en el balance de carbono de la planta (Flexas et al. 2006), condición que sería provocada en una primera instancia, por la disminución en la tasa de fotosíntesis neta debido a un descenso de la conductancia estomática. Para L. caustica, el proceso ocurrió cuando el contenido hídrico del sustrato se situó por debajo del $30 \%$ (día 82). Luego de este periodo ambos tratamientos recuperaron las tasas de crecimiento, siendo similares a las observadas en las plantas con riego permanente.

Respecto al crecimiento en altura, los resultados concuerdan con lo mencionado por Fotelli et al. (2000) para cuatro especies mediterráneas del género Quercus, concluyendo que la restricción hídrica no tendría incidencia en el incremento en altura. En el ensayo, esta situación fue atribuida a que el crecimiento de $L$. caustica es tanto horizontal como vertical, presentando a veces un crecimiento mayor en las ramas de la sección horizontal que en el eje principal.

En diferentes estudios, realizados con plantas bajo condiciones de déficit hídrico, se observa una disminución en el cociente biomasa aérea/biomasa radical, ocasionada por un mayor crecimiento del sistema de raíces (McMillin y Wagner 1995). En L. caustica, la biomasa radical de plantas severamente estresadas prácticamente igualó a la biomasa radical obtenida en plantas regadas permanentemente, no encontrando diferencias significativas en el crecimiento del sistema radical, entre tratamientos. El mismo comportamiento es observado para $A$. caven, no presentando diferencias significativas en cuanto a la biomasa de tallo y raíz (Donoso et al. 2015). En cambio, Q. saponaria presenta valores significativamente menores de biomasa radicular en plantas estresadas que en plantas no estresadas, bajo condiciones de ensayo similares (Donoso et al. 2015). Además, la biomasa total de $Q$. saponaria en plantas regadas es ampliamente superior a las plantas estresadas, situación que no ocurre en $L$. caustica, pues el valor de biomasa total en ambas condiciones fue prácticamente el mismo. Esto indicaría que $L$. caustica invierte una mayor proporción del crecimiento en sistema radical, desarrollando raíces finas aún bajo condiciones de estrés, permitiéndole acceder a nuevas fuentes de recurso hídrico y poblar sitios con clima mediterráneo. Parece, entonces, que $L$. caustica estaría mejor preparado para tolerar episodios de fuerte restricción hídrica que otras especies siempreverdes del bosque esclerófilo como $Q$. saponaria y $C$. alba.

Se ha señalado que en la región central de Chile, los procesos de reemplazo del bosque y matorrales esclerófilos están dando paso a procesos de recuperación por sucesión en campos abandonados (Hernández et al. 2016). Paralelo a esto, el cambio climático estaría aumentando los episodios más secos en esta región mediterránea (Peñuelas et al. 2004, Garreaud 2011). Los resultados logrados en este estudio muestran que $L$. caustica es una de las especies de mayor tolerancia al estrés hídrico entre las especies típicas, permitiendo entender mejor su establecimiento y desarrollo en los ambientes del bosque esclerófilo, en especial en etapas sucesionales tempranas. En este contexto, su papel en la recuperación del bosque esclerófilo puede ser muy importante. Además, al poner de manifiesto los mecanismos de esa tolerancia, estos resultados pueden potenciar las estrategias de manejo para la reforestación, tanto con técnicas de vivero como de campo (Vilagrosa et al. 2005, Cortina et al. 2006).

\section{CONCLUSIONES}

Como se esperaba, $L$. caustica muestra una notable tolerancia al estrés hídrico, que puede considerarse mayor a la encontrada en $Q$. saponaria y $C$. alba, pero menor a la de $A$. caven. Los resultados sugieren una buena respuesta de $L$. caustica a períodos de estrés hídrico, permitiendo a la especie ocupar sitios con menor disponibilidad de agua, establecerse temprano en la sucesión ecológica o permanecer en el tiempo ante una restricción del recurso. $\mathrm{La}$ especie desarrolla el mecanismo de ajuste osmótico, permitiendo mantener el turgor celular y un contenido hídrico relativo foliar alto (superior a $80 \%$ ). Esto explicaría su dominancia en la mayoría de zonas altas y de media altitud en la región mediterránea de Chile y su asociación con A. caven, especie pionera en la zona semiárida del país.

Cuando la restricción hídrica se hace severa, se produce el cierre estomático y una disminución en el rendimiento cuántico máximo del fotosistema II al mediodía, lo que trae consigo una disminución en la tasa fotosintética. Sin embargo, L. caustica es capaz de reparar los daños provocados durante el periodo de fotoinhibición observado a mediodía.

La reducción en el balance de carbono de la planta, provocada por la disminución en la tasa de fotosíntesis neta y el descenso de la actividad estomática, produce una reducción en la tasa de crecimiento en diámetro bajo condiciones de restricción hídrica moderada, y una detención cuando la restricción hídrica es severa. El crecimiento en altura no mostró un patrón claro, deficiencia que podría mejorarse en futuras evaluaciones aplicando una metodología de evaluación diferente a la presentada en este estudio.

Las plantas de L. caustica bajo estrés disminuyen el crecimiento foliar pero mantienen el crecimiento radical, lo que no ocurre en $Q$. saponaria y $C$. alba bajo condiciones similares. Independiente de la condición hídrica del suelo, existe una inversión constante de formar nuevas raíces para acceder a nuevas fuentes de recurso hídrico, condición que predispone a $L$. caustica a estar mejor preparado para tolerar los episodios de fuerte restricción hídrica.

\section{AGRADECIMIENTOS}

Este trabajo contó con el patrocinio del Programa Domeyko de Biodiversidad de la Universidad de Chile y del Programa de Bosques Mediterráneos de la Facultad de Ciencias Forestales y Conservación de la Naturaleza de la Universidad de Chile. En especial agradecer el apoyo del Dr. Juan Silva en la revisión del documento y por sus recomendaciones. 


\section{REFERENCIAS}

Cabello A, C Donoso. 2006. Lithraea caustica (Molina) Hook. et Arn. In Donoso C ed. Las especies arbóreas de los bosques templados de Chile y Argentina. Autoecología. Valdivia, Chile. Marisa Cuneo. p. 333-339.

Ceacero C, J Díaz-Hernández, A del Campo, R Navarro-Cerrillo. 2012. Evaluación temprana de técnicas de restauración forestal mediante fluorescencia de la clorofila y diagnóstico de vitalidad de brinzales de encina (Quercus ilex sub. ballota). Bosque 33(2): 191-202. DOI: 10.4067/S071792002012000200009

Corcuera L, J Camarero, E Gil-Pelegrín. 2002. Functional groups in Quercus species derived from the analysis of pressurevolume curves. Trees 16(7): 465-472. DOI: 10.1007/ s00468-002-0187-1

Cortina J, RM Navarro, A del Campo. 2006. Evaluación del éxito de la reintroducción de especies leñosas en ambientes mediterráneos. Capítulo 1. In Cortina J, JL Peñuelas, J Puértolas, A Vilagrosa, R Savé eds. Calidad de planta forestal para la restauración en ambientes Mediterráneos. Estado actual de conocimientos. Madrid, España. Organismo Autónomo Parques Nacionales. Ministerio de Medio Ambiente. p. 11-29.

Donoso C. 1982. Reseña ecológica de los bosques mediterráneos de Chile. Bosque 4(2): 117-146.

Donoso S, K Peña-Rojas, C Pacheco, G Luna, A Aguirre. 2011. Respuesta fisiológica y de crecimiento en plantas de Quillaja saponaria y Cryptocarya alba sometidas a restricción hídrica. Bosque 32(2): 187-195. DOI: 10.4067/S071792002011000200009

Donoso S, K Peña-Rojas, C Pacheco, S Durán, R Santelices, C Mascaró. 2015. The physiological and growth response of Acacia caven under water stress and the application of different levels of biosólidos. Ciencia e Investigación Agraria 42(2): 273-283. DOI: 10.7764/rcia.v42i2.1516

Fan S, T Blake, E Blumwald. 1994. The relative contribution of elastic and osmotic adjustments to turgor maintenance of woody species. Physiologia Plantarum 90(2): 408-413. DOI: 10.1111/j.1399-3054.1994.tb00406.x

Fleck I, K Peña-Rojas, X Aranda. 2010. Mesophyll conductance to $\mathrm{CO}_{2}$ and leaf morphological characteristics under drought stress during Quercus ilex L. resprouting. Annals of Forest Science 67(3): 308. DOI: 10.1051/forest/2009114

Flexas J, J Bota, J Galmés, H Medrano, M Ribas-Carbó. 2006. Keeping a positive carbon balance under adverse conditions: responses of photosynthesis and respiration to water stress. Physiologia Plantarum 127(3): 343-352. DOI: 10.1111/j.1399-3054.2006.00621.x

Flexas J, A Diaz-Espejo, J Gago, A Gallé, J Galmés, J Gulías, H Medrano. 2014. Photosynthetic limitations in Mediterranean plants: a review. Environmental and Experimental Botany 103: 12-23. DOI: 10.1016/j.envexpbot.2013.09.002

Fotelli M, K Radoglou, H Constantinidou. 2000. Water stress responses of seedlings of four Mediterranean oak species. Tree Physiology 20(16): 1065-1075. DOI: 10.1093/treephys/20.16.1065

Gallé A, P Haldimann, U Feller. 2007. Photosynthetic perfor- mance and water relations in young pubescent oak (Quercus pubescens) trees during drought stress and recovery. New Phytologist 174(4): 799-810. DOI: 10.1111/j.14698137.2007.02047.x

Galmés J, J Flexas, R Savé, H Medrano. 2007. Water relations and stomatal characteristics of mediterranean plants with different growth forms and leaf habits: responses to water stress and recovery. Plant Soil 290(1): 139-155. DOI: 10.1007/s11104-006-9148-6

Garreaud R. 2011. Cambio climático: Bases físicas e impactos en Chile. Revista Tierra Adentro 93: 13-19.

Grassi G, F Magnani. 2005. Stomatal, mesophyll conductance and biochemical limitations to photosynthesis as affected by drought and leaf ontogeny in ash and oak trees. Plant, Cell and Environment 28(7): 834-849. DOI: $10.1111 / \mathrm{j} .1365-$ 3040.2005.01333.x

Hernández A, M Miranda, E Arellano, C Dobbs. 2016. Landscape trajectories and their effect on fragmentation for a Mediterranean semi-arid ecosystem in Central Chile. Journal of Arid Environments 127: 74-81. DOI: 10.1016/j.jaridenv.2015.10.004

Krause G, E Weis. 1991. Chlorophyll fluorescence and photosynthesis: the basics. Annual review of plant biology 42(1): 313-349. DOI: doi/10.1146/annurev.pp.42.060191.001525

Luebert F, P Pliscoff. 2006. Sinopsis bioclimática y vegetacional de Chile. Santiago, Chile. Editorial Universitaria. 316 p.

Maxwell K, G Johnson. 2000. Chlorophyll fluorescence-a practical guide. Journal of experimental botany 51(345): 659668. DOI: $10.1093 /$ jexbot $/ 51.345 .659$

McMillin J, M Wagner. 1995. Effects of water stress on biomass partitioning of Ponderosa Pine seedlings during primary root growth and shoot growth periods. Forest Science 41(3): 594-610.

Peña-Rojas K, X Aranda, I Fleck. 2004. Stomatal limitation to $\mathrm{CO}_{2}$ assimilation and down-regulation of photosynthesis in Quercus ilex resprouts in response to slowly imposed drought. Tree Physiology 24(7): 813-822. DOI: 10.1093/ treephys/24.7.813

Peñuelas J, S Sabaté, I Filella, C Gracia. 2004. Efectos del cambio climático sobre los ecosistemas terrestres: observación, experimentación y simulación. In Valladares F ed. Ecología del bosque mediterráneo en un mundo cambiante. Ministerio de Medio Ambiente. Madrid, España. EGRAF S. A. p. $425-460$.

Root-Bernstein M, R Valenzuela, M Huerta, J Armesto, F Jaksic. 2017. Acacia caven nurses endemic sclerophyllous trees along a successional pathway from silvopastoral savanna to forest. Ecosphere 8(2): e01667.10.1002/ecs2.1667. DOI: $10.1002 /$ ecs 2.1667

Tyree M, H Richter. 1981. Alternative methods of analysing wáter potential isotherm: some caution and clarifications. Journal of Experimental Botany 32(128): 643-653. DOI: 10.1093/jxb/32.3.643

Vilagrosa A, J Cortina, E Rubio, R Trubat, E Chirino, E Gil-Pelegrin, VR Vallejo. 2005. El papel de la ecofisiología en la restauración forestal de ecosistemas mediterráneos. Investigación Agraria. Sistemas y Recursos Forestales 14(3): 446-461. 